\title{
PROFESSIONALS' OPINIONS ON MULTIDIMENSIONAL APPROACH TO EARLY INTERVENTION FOR CHILDREN WITH ASD
}

\author{
Eleni-Anna Kardara \\ European University of Cyprus, Greece \\ Nanou Andromachi \\ Scientific Collaborator of European University of Cyprus, Greece
}

\begin{abstract}
Early intervention methods for children with Autism Spectrum Disorders, increasingly turn to multidimensional approaches with a parallel focus on interprofessional collaboration in order the interventions to be effective. Multidimensional approaches efficiently support the complex system of family interaction and function affecting positively the child's development. Early and efficient interventions are widely recognized to lead to long-term positive socio-cognitive results for children with Autism Spectrum Disorders and their families. The purpose of this research is to delve into professionals', special educators 'and therapists', opinions on their collaboration with parents and other professionals as they apply early intervention programs to children with ASD. Semi-structured interviews of 15 professionals, experts on early intervention in children with ASD, from different regions of Greece, were analyzed using qualitative research methods. Part of the data analysis that presented in this paper demonstrates clearly that the professionals' and special educators' opinions emphasize the need for multidimensional approaches. The analysis also highlights the obstacles that restrict collaboration with parents and other professionals. The material resources of state support have also been suggested as factors that interact with family resources that affect the child's developmental characteristics.
\end{abstract}

Keywords: autism, ASD, early intervention, interprofessional collaboration, multidimensional approach, parents.

\section{Introduction}

Autism Spectrum Disorder (ASD) is a complex, lifelong neurological and developmental disorder, whose etiology continues to be investigated. Early intervention leads to long-term positive results for children with ASD and promises to reduce syndrome's imprint. Professionals and special educators' emphasize the need for multidimensional approaches in early intervention that include the child itself, parents, siblings, and peer's (Simpson, de Boer-Ott, \& Smith-Myles, 2003). 
Kardara \& Andromachi, 2021. Professionals’ Opinions on Multidimensional Approach to Early Intervention for Children with ASD

Multidimensional approaches have been highlighted by the World Health Organization. Specifically, the WHO's "Framework for Action on Interprofessional Education and Collaborative Practice" (Health Professions Network Nursing and Midwifers office within the Department of Human Resuourses for Health, 2010) emphasizes the need for multidimensional collaboration. Collaboration is not only a point of consensus among professionals, but it is also related to their collaboration and creativity. Synergy can develop between them when those included- interact closely and have a shared understanding of their field, and results that demonstrate progress in their knowledge regarding the child's treatment and care (Health Professions Network Nursing and Midwifers office within the Department of Human Resources for Health, 2010). Interprofessional collaboration is defined as prerequisite by the United Nations Convention for the Rights of persons with Disabilities and emphasized in the National Institute for Health and Care excellence in guidance materials (Dillenburger et al., 2014). The Autism Spectrum Disorder Inclusion Collaboration Model (ASDICM) that was developed by Simpson and his colleagues promotes the cooperation between educators, parents, and caregivers' as part of their assignment, to teach and advocate for children with ASD (Simpson, de Boer-Ott, \& Smith-Myles, 2003). The underlying principle of this model is cooperation between participants, so they can promote an individualized educational program (IEP), which can be characterized as a complete course based on a child's specific needs (Simpson, de Boer-Ott, \& Smith-Myles, 2003). The ASDICM is made up of five elements: a) changes to the surroundings and educational program, b) behavioral and social support, c) efficient team engagement, d) evaluation of the process of inclusion and e) home- school cooperation (Simpson, de Boer-Ott, \& Smith-Myles, 2003).

Furthermore, frameworks like the ASDICM, inspire confidence in specialized educators and services, so that they can provide and share evidencebased practices and models. The ASDICM attaches particular importance to the role of parents throughout an intervention (La Barbera, 2017). Also, as Anthony \& Campbell have underlined, in addition to the special educators, therapists, and parents, social workers and also valuable experts, who can provide valuable knowledge and skills from their own field (Anthony \& Campbell, 2020). As all the parts come together, the communication between them can guide them to create a mutual space of understanding and promoting shared goals (Avendano, \& Cho, 2020). Avendano \& Cho (2020) highlight the impact of the Individuals with Disabilities Education Improvement Act (2004). IDEA (IDEA, 2011) recognizing the importance of the parents' involvement in their child's education. IDEA indicates that schools must collaborate with the parents of children with disabilities and support parents and families. Some of the guidelines of IDEA are the following: all schools must maintain an open communication with the parents. 
Parents should also be involved in the planning of interventions related to their child and should receive help in developing their own skills so that they can participate in the education of their child (Avendano, \& Cho, 2020).

In Julie Strunk, Melissa Leisen and Carolyn Schubert's (2017) in their international systematic review, they reviewed 47 articles in which they explored the use of multidimensional collaboration in cases with children with ASD (Strunk, Leisen, \& Schubert, 2017). Their conclusions showed that there are only a few sectors that can use a multidisciplinary approach when working with kids with autism. One of the reasons why interprofessional and interdisciplinary teamwork doesn't happen is a lack of understanding of the roles and responsibilities of each agent. Lack of time and variable schedules are also cited a hindrance as is avoiding confrontation. Finally, the need for personal recognition is recognized as a particularly powerful factor (Strunk, Leisen, \& Schubert, 2017).

In order for collaboration between the members of the teams supporting to be achieved, five fundamentals are required: a) all members should be working towards a shared goal, b) all members should be valued for their unique views, d) all professions comprising the team must have a strong sense of purpose, and e) there should be a sense of trust and shared responsibility among team members (Strunk, Leisen, \& Schubert, 2017)

\section{The Main Purpose}

The main purpose of this study is to investigate the professionals' opinions regarding the effectiveness of early intervention methods for children 3-7 years old, with a special focus on collaboration with the other professionals and with the parents of children with ASD and with the school teachers. The study attempts to address the following research questions: a) What are the opinions of the professionals' concerning the interprofessional collaboration in early intervention for children with ASD? b) what are professionals' opinions concerning the contribution of parents and the collaboration with them during the implementation of early intervention programs?

\section{Participants}

15 early intervention professionals from Greece and Cyprus participated in this research. Multiple cities and geographic areas were represented. The majority (nine) 9 were from cities in Southern Greece, (two) 2 from Cyprus, (two) 2 from Northern Greece and (two) 2 from Crete. Ages ranged between 25-40. Their professionals' fields of expertise included: (seven) 7 phycologists, (two) 2 speech therapists, (four) 4 occupational therapists and 2 special educators. All participants worked in or run private early intervention centers. Such privately- 
Kardara \& Andromachi, 2021. Professionals’ Opinions on Multidimensional Approach to Early Intervention for Children with ASD

run early interventions are paid by parents, but are subsidized by the state after diagnosis from public hospitals and mental health centers and the recommendation for early intervention.

\section{Methodology}

The qualitative research methodology was chosen for this study in order for the professionals' personal views to be collected by interacting with them directly. Semi-structured interviews were designed as a qualitative data collection method to further investigate professionals' views delving into their experiences of children with ASD and their families through early interventions. The unstructured interview covered three major areas: i) opinions on early intervention methods for children with ASD, ii) opinions concerning the factors that affect the effectiveness of early interventions, iii) suggestions and best practices for the education and social inclusion of children with ASD.

As conditions did not allow for live interviews due to the COVID-19 pandemic, the participants were sourced and selected by putting out requests in social media and specifically in the professionals' Facebook Groups: "Autism Spectrum Disorders", "Special education in Greece”. The reasons for the request for participants and the objective of study was explained in a written message posted in those social media groups. After the participants had expressed their interest in the research, the interview protocol was sent via message to their personal profiles, in which more explanations were given about the research, its purpose and the research questions. Individual interviews were conducted by video call via the Skype or Messenger Applications and lasted for a maximum of 45 to 60 minutes. During the interviews the researcher kept personal notes and the interview was recorded with a digital audio recorder after obtaining consent from the participants "PHILIPS DVT2510 8GB Voice Tracer Audio Recorder Notes Recording". The atmosphere in the interviews is intimate, friendly and enthusiastic. The participants expressed their interest and preference for the qualitative research methodology and the choice of the specific topic and they really expressed their wish to help in any way that they could. The qualitative methodology in particular drew their interest, because they felt that it would be intriguing to see the views and concerns of their colleagues. The majority were very forthcoming in answering the questions and providing a wealth of detail, citing personal examples from their own experiences. When the interviews were over, the recording and coding took place in three stages, A to C.

In stage A fourteen (14) codes were developed. These codes in stage B were reduced to six (6), and at the final stage $C$ to three (3). At the final, higher-level three (3) codes were developed: "Early intervention effective methodologies", "Best practices for social inclusion of children with ASD" and "Obstacles". This 
paper presents the findings concerning the latter two (2) codes: "Best practices for social inclusion of children with ASD" and "Obstacles". At these codes the professionals' opinions on the interprofessional collaboration and the parents' contribution are included.

A narrative methodology was chosen for data analysis because this approach pays special attention to relations and consistency of the data. The main focus is on the aspect and conformation of the narrative. Narration can be examined as the way the narrator uses it in front of an audience (Tsiolis, 2015). The researcher used they/them pronouns for the fine-grained analysis of the data, concealing the participants' gender, which wasn't a factor in the analysis. The production process was chosen for analysis and coding pre-selecting conceptual schemes from the beginning of the research, which functioned as taxonomic guides for data organization (Tsiolis, 2015).

\section{Findings}

The professionals' opinions that was coded in stage $C$ at the last two codes "Best practices for social inclusion of children with ASD" and "Obstacles" are being presented in accordance with the research questions:

a) What are the professionals' opinions concerning the interprofessional collaboration in early intervention for children with ASD?

According to the coded statements of the participants under the title "Best practices for social inclusion of children with ASD”, all participants, speech therapist, occupational therapist, psychologists and special educators (15/15), emphasized the need of interprofessional collaboration and highlighted the parents' and all family support and also the collaboration with preschool teachers.

Also, the majority of participants (13/15) suggested that multidimensional approaches have better results for the children with ASD, because a multidimensional team of experts will prioritize the needs of the child, and adjust the goals, short-term or long-term, according to the results of a personal assessment. They also referred to the need for the complementary relationships between the professionals' the family and preschool teachers at schools in order for the early intervention program to be effective and social inclusion to be fostered. The majority of the participants supported the multidimensional approach to be established with the collaboration and open communication of the two relevant ministries, Health' and Educations'

Concerning the obstacles that restrict the effectiveness of early intervention programs in Greece the participants referred to managing the sense of authority of each specialist and the lack of time for the interprofessional collaboration. Most participants (12/15) said that there is no interprofessional cooperation happening because there is no time- as they try to balance different schedules. They 
Kardara \& Andromachi, 2021. Professionals’ Opinions on Multidimensional Approach to Early Intervention for Children with ASD

expressed that one of the factors that negatively affect collaboration is that there is not enough time for meetings between professionals or planning while taking all perspectives into account.

"I believe that it is not always possible for therapists to cooperate. Often the plans that are made to reach a goal do not include everyone's views, some may be omitted by either speech therapists or occupational therapists."

"It is very difficult to keep working with the team supporting the child, because there is no time. There are different schedules, and little time to get along. -"I have the feeling that they are not trying hard."

Additionally, four of the fifteen (4/15) participants underlined as an obstacle the lack of appreciation between early intervention team members and the trend of some professions undervaluing others.

"There is a lot of controversy in terms of the methods used, so there is confusion and there is no proper cooperation of therapists with each other. "Everyone tries to see how they will prevail over the other without a basic research in the literature."

Concerning the collaboration with preschool teachers the participants (8/15) insisted that collaboration with schools is not an easy task either, because in public schools, the training of preschool teachers is not sufficient on the treatment of autism spectrum disorders. Preschool teachers do not seek out opinions or cooperation from professionals and therapists so the skills that are being developed by the child during the individual therapies are difficult to be generalized. One of the participants referred that in some cases there is a wall between teachers and professionals:

"There are cases of good cooperation but they are minimal, many times the school as a unit puts up a wall in communication."

"There is no proper cooperation with the school either, because many teachers do not have the necessary education in autism, to have a general knowledge of the specific diagnostic category, the problem starts from there."

One of the reasons for that is that the school and therapists operating hours are different, which in itself poses barriers to communication.

"I do not have any good communication with the civil servants, because they only want to communicate during their working hours. They force you to speak harshly. The way the civil servant works is different from the private one".

b) What are professionals' opinions concerning the contribution of parents and the collaboration with them during the implementation of early intervention programs?

Concerning the collaboration between parents and professionals, the participants highlighted the need for truthfulness and close cooperation. Although they declared that they need parents' cooperation they admitted that, sometimes, 
they feel that the parents do not have the psychological strength to fully undertake to the child's support and monitoring requirements. The family of the disabled child faces a lot of stressors, as it is broadly recognized by all the participants. All the professionals argued that the parents need to be supported by mental health professionals, especially when their child initially gets the ASD diagnosis, and start a program of consultation supporting them in dealing with the process of grief and the backlash that they are experiencing.

The majority of the participants insist that the parents have to take time and space to deal with the diagnosis, because if parents are forced to work with the child the result will not be sufficient. Then the parents may stop trusting the therapist so they are not willing enough to offer their observations and feedback.

Although the majority of participants praise the results of cooperation between parents and therapists, most of them (ten of fifteen, 10/15) recognized a lot of difficulties. For example, they mention:

"Although the parents are happy about the abilities they establish with the child, they don't want to cooperate with the professional through feedback, so the child wigwag"

Most professionals interviewed believe that the parents' involvement is important only in order for the effects of the intervention to be generalized in context beyond the therapeutic center. But the multiple factors make that collaboration harder:

"Maybe the parents are not familiar with the intervention procedures, or they can't imagine what the child needs because of their position as parents, this is the part that the professional works like a bridge of communication between them, and they help both parts to make a connection."

Many therapists also referred to the cost of the therapies as an obstacle for parents to choose extensive and multifaceted treatments. They highlighted that the cost of the early intervention programs is high, and the economic support from the state is minimal. Due to this reason most parents choose only the minimum of the needed treatments prescribed as part of the medical release. This cost in accordance with the lack of full economic support from the state was referred by most participants as the biggest obstacle for the execution of the multidimensional intervention that promotes collaboration with other professionals and the support of all the members of a child's family and its preschool teachers at kindergartens.

\section{Research Findings / Discussion}

The multidimensional approach seems an ideal that all professionals support. Professionals interviewed agree with the importance of interprofessional collaboration and on the values that must be present among the team members. Our research findings also agree on the specific values that characterize teamwork 
Kardara \& Andromachi, 2021. Professionals’ Opinions on Multidimensional Approach to Early Intervention for Children with ASD

and that the main purpose of early intervention by interprofessional teams has to be the support of the child with ASD and its family. This finding agrees with the one of the research conducted by Julie Strunk, Melissa Leise, and Carolyn Schubert (2017).

Although professionals believe in the importance and effectiveness of the multidimensional approach, a lot of obstacles arise in practice as the research participants indicate. The opinions of the participants are in agreement with the research of Strunk and colleague. According to both research findings, a major reason why interprofessional team work doesn't happen as effectively as it should is a lack of understanding of the roles and responsibilities in those teams. Another reason is the lack of time and different schedules, in addition to the avoidance of confrontation and the need for personal recognition (Strunk, Leisen, \& Schuber , 2017).

Concerning the views of participants about collaboration with parents, a very important finding is that professionals view parental involvement as a necessary for generalizing the results of the intervention at school and at home. None of the therapists focused on parental involvement in planning interventions and none talked about them taking an active role during treatment. Collaboration difficulties between parents and therapists or disagreements on how to proceed with the child that are referred to as obstacles in early intervention effectiveness shows that professionals need support to involve parents of children with ASD in the therapeutic process. According to the guidelines of IDEA, parents must be involved in the planning of their child's interventions, and parents must receive help in the development of the skills that will allow them to participate in the education of their child. Parents' role is crucial in the designing the IEP plan (Avendano, \& Cho, 2020).

Finally, the majority of participants supported the multidimensional approach to be established with the collaboration of and close communication between the ministries of Health and Education. For the context of Greece specifically, this is evaluated as potentially significant for early intervention.

\section{Limitations.}

The $15^{\text {th }}$ participants in this research express their thoughts and views on how the early intervention and the methods we use can be more efficient. Our study has a strength in the nationwide distribution of informants as the professionals were from different areas of Greece. However, their personal interest and views can only express themselves and not how the things are handled in Greece.

Future Research.

In further research, qualitative research methodology could be applied in investigating how the multidimensional approach going on in Greece and Europe. 


\section{Acknowledgements}

This work was conducted while the author was a student at European University of Cyprus as part of completing her MSc thesis.

\section{References}

Anthony, N., \& Campbell, E. (2020). Promoting Collaboration Among Special Educators, Social Workers, and Families Impacted by Autism Spectrum Disorders. Advances in Neurodevelopmental Disorders. DOI:10.1007/s41252-020-00171-w

Avendano, S., \& Cho, E. (2020). Building Collaborative Relationships With Parents. TEACHING Exceptional Children, 52(4), 250-260. DOI:10.1177/0040059919892616

Dillenburger, K., Rottgers , H.-R., Douvani, K., Sparkman, C., Keenan, M., Thyer, B., \& Nikopoulos, C. (2014). Multidisciplinary Teamwork in Autism: Can one size fit all? The Australian Educational and Developmental Phycologist, 31(2), 97-112. DOI:10.1017/edp.2014.13

Health Professions Network Nursing and Midwifers office within the Department of Human Resuourses for Health. (2010). Framework for action on interprofessional Education \& Collaborative Practices. World Health Organisation .

IDEA. (2011). IDEA 2004 Summary. Retrieved from https://web.archive.org/web /20111027180848 /http://www.fape.org/idea/2004/summary.htm

La Barbera, R. (2017). A Comparison of teacher and caregiver perspectives of collaboration in the education of students with autism spectrum disorders. Teacher Education Quarterly, 44(3), 35-56.

Simpson, R., de Boer-Ott, S., \& Smith-Myles, B. (2003). Inclusion of Learners with Autism Spectrum Disorders in General Education Settings. 116-133. Lippincott Williams \& Wilkins, Inc. .

Strunk, J., Leisen, M., \& Schubert, C. (2017). Using a multidisciplinary approach with children diagnosed with autism spectrum disorder. Journal of Interprofessional Education \& practice, 60-68. DOI:10.1016/j.xjep.2017.03.009

Tsiolis, G. (2015). Analysis of qualitative of data collections: dilemmas, opportunities, abilities, procedures. 